\title{
Raise a glass to vascular health
}

It's no secret that vascular health declines with age. Contributing to this decline is dysfunction of the endothelium, the inner lining of the blood vessels. Normally, a functional endothelium enables coagulation, platelet adhesion and immune activity. However, its dysfunction is associated with reduced anticoagulant properties and an inability of arteries and arterioles to dilate fully, which contribute to the development of various diseases, especially cardiovascular disease. Endothelial dysfunction also reduces exercise capacity with age.

Previous studies have suggested that dietary intake of polyphenols (compounds found in fruit- and vegetable-derived foods and beverages, including red wine) lessens the risk of cardiovascular disease and that this effect may be exerted through direct action of polyphenols on endothelial cells.

In a new study published in Biochemical and Biophysical Research Communications (404, 743-749; 2011), Valérie B. Schini-Kerth (Université de Strasbourg, Illkirch, France) and colleagues examined whether red wine

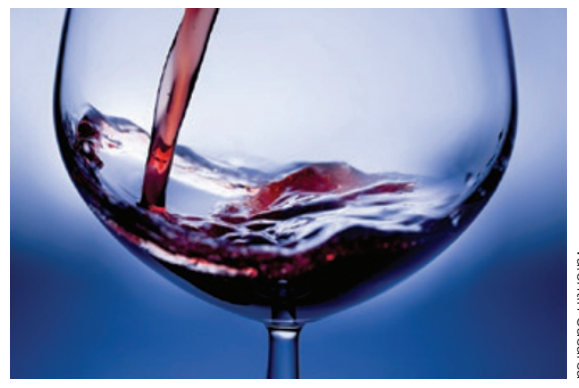

This work suggests that intake of red wine polyphenols prevented aging-related endothelial dysfunction and also improved the exercise capacity of aged rats. These benefits seem to involve the ability of red wine polyphenols to reduce oxidative stress by inhibiting the enzyme NADPH oxidase, which is associated with the hardening of arteries and an increased risk of heart disease. According to the authors, "[t]he beneficial effect of [red wine polyphenols] is explained best by their ability to maintain oxidative stress in the old arterial wall to a level similar as that observed in young arteries."

In this study, red wine polyphenols were administered to rats that were relatively young. In a review of the study, independent experts from the International Scientific Forum on Alcohol Research note that some previous studies suggest that interventions to improve endothelial function (such as consumption of polyphenols) should begin early in life to slow aging-related endothelial dysfunction. These results tend to support this notion.

Monica Harrington

\section{SICK WORMS HELP ELUUCIDATE VIRAL_INFECTIONS}

For the first time, researchers have identified viruses that naturally infect nematodes in the wild (PLoS Biol. 9, e1000586; 2011). Though scientists have extensively studied bacterial infections in the nematode Caenorhabditis elegans, they have only been able to carry out limited studies of antiviral responses in this microscopic worm. Scientists will now be able to use these viruses to study host-virus interactions in nematodes, which could lead to the identification of alternative tactics for fighting off viral infections.

Marie-Anne Félix of the CNRS-University of Paris-Diderot in France and colleagues isolated infected C. elegans and C. briggsae, a related worm, from rotting fruit. The infected worms did not get better after receiving antibiotics. The researchers homogenized tissue from infected worms and filtered this solution, leaving out any particles larger than a virus. These filtered homogenates infected healthy worms, leading Félix and colleagues to suspect that a virus might have been causing the infections.

Further analyses lead to the identification of two novel RNA viruses, one infecting C. elegans (Orsay virus) and the other infecting C. briggsae (Santeuil virus). These viruses are distantly related to known viruses in the family Nodaviridae, which infect fish and insects. The two novel viruses share only about $40 \%$ of their genetic material with previously identified nodaviruses. Orsay virus and Santeuil virus share a novel genetic sequence, called ORF $\delta$, which is not found in any of the previously identified nodaviruses. The authors suspect that the protein encoded by ORF $\delta$ might play an important role in the viruses' ability to infect or replicate in nematodes.

Researchers can use a process called RNA interference (RNAi) to systematically block the expression of almost every gene in the genome of $C$. elegans. Some plants and animals use RNAi to target and silence gene expression of invading viruses. Félix and colleagues found that a strain of mutant $C$. elegans that is deficient in the RNAi pathway accumulated more viral RNA than did the commonly used wild-type strain of $C$. elegans.

These results indicate that the RNAi pathway likely plays a role in defending worms against Orsay virus infection. Study of the genetic differences between strains of $C$. elegans that have different levels of susceptibility to infection by the Orsay virus might lead to the identification of genes involved in halting the spread or replication of the virus. Results from such studies could help researchers develop antiviral treatments for humans. 